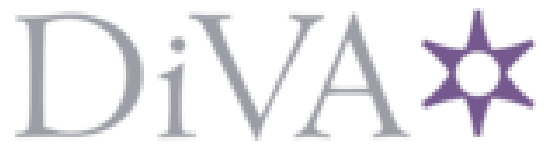

http://www.diva-portal.org

This is the published version of a paper published in Journal of Chemical Physics.

Citation for the original published paper (version of record):

Emanuelsson, C., Johansson, L., Zhang, H. (2018)

Delicate Interactions of PTCDI molecules on $\mathrm{Ag} / \mathrm{Si}(111)-\sqrt{ } 3 \times \sqrt{3}$

Journal of Chemical Physics, 149(16): 164707

https://doi.org/10.1063/1.5053606

Access to the published version may require subscription.

N.B. When citing this work, cite the original published paper.

Permanent link to this version:

http://urn.kb.se/resolve?urn=urn:nbn:se:kau:diva-70246 
Delicate interactions of PTCDI molecules on $\mathrm{Ag} / \mathrm{Si}(111)-\sqrt{3} \times \sqrt{3}$

C. Emanuelsson, L. S. O. Johansson, and H. M. Zhang

Citation: The Journal of Chemical Physics 149, 164707 (2018); doi: 10.1063/1.5053606

View online: https://doi.org/10.1063/1.5053606

View Table of Contents: http://aip.scitation.org/toc/jcp/149/16

Published by the American Institute of Physics

\section{PHYSICS TODAY}

WHITEPAPERS
ADVANCED LIGHT CURE ADHESIVES

READ NOW

Take a closer look at what these environmentally friendly adhesive systems can do
PRESENTED BY

(8) MASTERBOND' 


\title{
Delicate interactions of PTCDI molecules on $\mathrm{Ag} / \mathrm{Si}(111)-\sqrt{3} \times \sqrt{3}$
}

\author{
C. Emanuelsson, ${ }^{\text {a) }}$ L. S. O. Johansson, and H. M. Zhang \\ Department of Engineering and Physics, Karlstad University, SE-651 88 Karlstad, Sweden
}

(Received 23 August 2018; accepted 8 October 2018; published online 24 October 2018)

\begin{abstract}
PTCDI molecules were evaporated onto a $\mathrm{Ag} / \mathrm{Si}(111) \sqrt{3} \times \sqrt{3}$ surface and studied using scanning tunneling microscopy (STM) and angular resolved ultraviolet photoelectron spectroscopy (ARUPS). High resolution STM images are used to identify the delicate molecule/molecule and molecule/substrate interactions and the shapes of the molecular orbitals. The results show that the substrate/molecule interaction strongly modifies the electronic configuration of the molecules as their orbital shapes are quite different at 1 and 2 monolayer (ML) coverage. Simple models of molecular HOMO/LUMO levels and intermolecular hydrogen-bondings have been made for 1 and 2 ML PTCDI coverages to explain the STM images. Changes due to the interaction with the substrate are also found in ARUPS as extra states above the regular HOMO level at 1 ML PTCDI coverage. The ARUPS data also show that the electronic structure of the substrate remains unchanged after the deposition of molecules as the dispersion of the substrate related bands is unchanged. The changes in electronic structure of the molecules are discussed based on a HOMO/LUMO split. Published by AIP Publishing. https://doi.org/10.1063/1.5053606
\end{abstract}

\section{INTRODUCTION}

Organic semiconductors and the application of these electronic devices have attracted great attention from the scientific community over the last decades. These efforts have resulted in several promising applications such as light emitting diodes,${ }^{1}$ field effect transistors, ${ }^{1,2}$ solar cells, ${ }^{1,3}$ photodetectors, ${ }^{3}$ and organic lasers. ${ }^{4}$ Many of these devices involve organic thin films, hence their electronic properties and performance are highly dependent on the quality of the films. The structure and quality of the films are strongly influenced by the structural order of the first monolayers (MLs) of the films. The interface between organic and inorganic materials is therefore of special interest. The formation of the interface involves interactions between molecules within the molecular film and interactions between the film and the substrate. A detailed understanding of these delicate interactions may therefore be used to optimize organic-inorganic devices.

The perylene derivative 3,4,9,10-perylene tetracarboxylic dianhydride (PTCDA) has been used as a model molecule to study the growth of organic films on a variety of substrates. ${ }^{5}$ The intermolecular interactions in PTCDA films are governed by hydrogen $(\mathrm{H})$-bondings between the oxygen atoms in the carboxylic anhydride endgroup and the $\mathrm{H}$ atoms on the sides of the perylene core. To further understand these intermolecular interactions and their importance in thin film formation, one could consider similar molecules but with a different endgroup. This should give rise to different possibilities to form intermolecular H-bondings. A good candidate in this respect is 3,4,9,10-perylene tetracarboxylic

a)christian.emanuelsson@kau.se diimide (PTCDI). The only difference from PTCDA is that the bridging oxygen atom in the carboxylic anhydride group is substituted with $\mathrm{N}-\mathrm{H}$ in PTCDI. The strongest intermolecular interaction for PTCDI is the $\mathrm{N}-\mathrm{H} \cdots \mathrm{O} \mathrm{H}$ bonding between the endgroups of neighbouring molecules. Three phases of PTCDI have so far been found experimentally, namely, brick wall, ${ }^{6,7}$ domino, ${ }^{7}$ and canted phases. ${ }^{6-11}$ These three phases all involve the aforementioned $\mathrm{N}-\mathrm{H} \cdots \mathrm{O}$ $\mathrm{H}$-bondings but are also further stabilized by extra $\mathrm{O} \cdots \mathrm{H}$, H-bondings. Understanding the interplay between surface periodicity, strength of intermolecular, and substrate/molecule interactions is therefore of great importance for utilizing this family of molecules to create ordered layers or even nanostructures such as $1 \mathrm{D}$ rows or supermolecular surface assemblies. ${ }^{12,13}$

We have previously studied PTCDI on $\mathrm{Ag} / \mathrm{Si}(111)$ $\sqrt{3} \times \sqrt{3}$ using scanning tunneling microscopy/spectroscopy $(\mathrm{STM} / \mathrm{STS})^{11}$ and photoemission. ${ }^{14}$ This surface is a metalinduced reconstruction that is weakly interacting, and therefore a suitable substrate for growing self-assembled organic films. The surface is composed of $\mathrm{Si}$ - and Ag-trimers and has a hexagonal structure described by the inequivalent triangle model, which has a semiconducting characterization. ${ }^{15}$ On this surface, the molecules arrange themselves according to the aforementioned canted structure: Molecules grow in rows and are tilted slightly different in every other row. The growth of the first monolayer involves a superstructure which can be seen in STM as rows of darker molecules. This superstructure has a $3 \sqrt{39} \times \sqrt{21}$ periodicity relative to the $\mathrm{Ag} / \mathrm{Si}(111)-\sqrt{3} \times \sqrt{3}$ and includes 5 regular molecular unit cells. The photoemission study showed extra features at coverages up to $1 \mathrm{ML}$ in $\mathrm{C}, \mathrm{O}$, and $\mathrm{N} 1 \mathrm{~s}$ core-level spectra. These extra features are located at lower binding energies relative to the main features of each spectrum. The near edge X-ray absorption fine 
structure (NEXAFS) spectra of the C, O, and N K-edges show that the first transitions to the LUMO are less intense for coverages below $1 \mathrm{ML}$, and the ultraviolet photoelectron spectroscopy (UPS) spectra below $1 \mathrm{ML}$ present extra features between the normal HOMO and the Fermi level.

These changes were explained by the interaction between the molecules and the substrate resulting in two types of molecules. The two types of molecules have their own HOMO and LUMO pairs but shifted in energy positions. In the previous study, there was also a discrepancy when comparing UPS and STS spectra: At 1 ML coverage, the UPS spectra have a state at $1 \mathrm{eV}$ below the Fermi level, which did not appear in the STS spectra. In this paper, we have studied PTCDI on $\mathrm{Ag} / \mathrm{Si}(111)-\sqrt{3} \times \sqrt{3}$ using high resolution STM images to figure out the molecular configurations and deeply investigated the molecule-molecule interactions. We also used high resolution angular resolved ultraviolet photoelectron spectroscopy (ARUPS) to investigate the electronic structure between the HOMO and the Fermi level to find out the origin of the states at 1 and 2 ML PTCDI coverages.

\section{EXPERIMENTAL DETAILS}

The STM study was performed with an Omicron STM system at Karlstad University using a W/Ir tip. The ARUPS studies were performed at beamline $\mathrm{I} 4^{16,17}$ at the MAX-lab synchrotron facility located in Lund, Sweden. The valence band data were measured with energy and angular resolution of $50 \mathrm{meV}$ and $\pm 0.3^{\circ}$, respectively. The samples used were Shiraki-etched ${ }^{18} \mathrm{Si}(111)$, Sb-doped to a resistivity of $1-10 \Omega \mathrm{cm}$. The oxide of the sample was removed by stepwise resistant heating up to $940{ }^{\circ} \mathrm{C}$, resulting in a high quality $\mathrm{Si}(111)-7 \times 7$ surface. The $\mathrm{Ag} / \mathrm{Si}(111)-\sqrt{3} \times \sqrt{3}$ reconstruction was created by evaporating $1 \mathrm{ML} \mathrm{Ag}$ onto the substrate and annealing it at $600^{\circ} \mathrm{C}$ for $2 \mathrm{~min}$. In both STM and photoemission experiments, low energy electron diffraction (LEED) investigations of the surface showed a sharp $\sqrt{3} \times \sqrt{3}$ diffraction pattern. PTCDI was then evaporated using a Knudsen cell held at $275^{\circ} \mathrm{C}$ while the sample was kept at room temperature (RT). STM images were recorded at several PTCDI coverages ranging from sub- up to $5 \mathrm{ML}$, while the valence band spectra were recorded on a clean substrate, $1 \mathrm{ML}$ and 2 ML PTCDI coverages. All measurements were done with the sample at RT, and all STM bias voltages in the paper were sample-biased.

\section{RESULTS AND DISCUSSION}

As shown in the previous studies, PTCDI grows in wellordered layers on the $\mathrm{Ag} / \mathrm{Si}(111)-\sqrt{3} \times \sqrt{3}$ surface. ${ }^{9,11}$ The only phase that was found is the canted structure. One example of such a structure in a PTCDI island recorded at two different tunneling biases is presented in Fig. 1. The unit cell of the canted structure contains two molecules and is marked with a small rectangle in both images in Fig. 1. Figure 1(a) is a filled-state image recorded at $-1.0 \mathrm{~V}$, while Fig. 1(b) is an empty-state image recorded at $1.0 \mathrm{~V}$. In both images, a clear intensity difference between different molecules is evident as some molecular contrasts in brightness are opposite
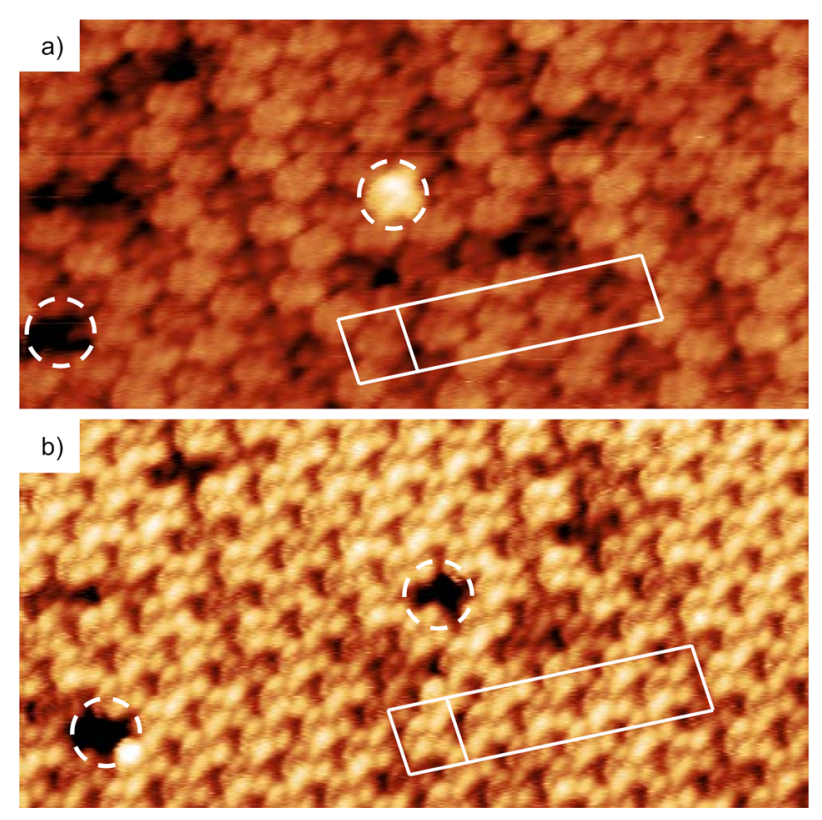

FIG. 1. A 1 ML PTCDI island recorded at different biases of (a) $-1.0 \mathrm{~V}$ and (b) $1.0 \mathrm{~V}$. In each image, two defects have been marked with circles. The left is a vacancy while the right one is a molecule. The unit cell of the canted structure and the super structure is also marked with rectangles in each image. Each image is $20 \times 10 \mathrm{~nm}^{2}$ and recorded using $\mathrm{I}=0.3 \mathrm{nA}$.

in the filled and the empty-state. Rows of molecules that are less intense form a superstructure with a $3 \sqrt{39} \times \sqrt{21}$ periodicity. This superstructure is marked with longer rectangles in Fig. 1. The superstructure shows that the canted structure has a commensurate relation to the substrate which implies that the PTCDI molecules interact weakly with the substrate and form long-range orderings. In both Figs. 1(a) and 1(b), the unit cell for the superstructure has been drawn on the same molecules as can be verified by using the two defects marked with circles in both images. The superstructure is clearly observable in both biases as rows of less intense molecules, but interestingly, the molecules that appear less intense are different in the two images. In Fig. 1(a), the corner molecules of the superstructure are brighter while they appear darker in 1(b). This variation in intensity among molecules at different tunneling biases shows that molecules in the film have different electronic structures.

A set of high resolution filled-state images of molecules in a $1 \mathrm{ML}$ island at three different tunneling biases are shown in Figs. 2(a)-2(c). Using these images, it is possible to determine how the orientation and location of the orbital lobes change with tunneling biases. The lobes that are observed in each STM image have been drawn onto stick and ball models of the PTCDI molecule. These configurations are presented under each respective STM image in Fig. 2. In all three cases, four of the lobes are located at the hydrogen atoms at the edges of the molecules. The other lobes are located at the perylene core of the molecule distributed along the main axis. However, such appearances of the molecule are unexpected considering that in single molecular density functional theory (DFT) calculations the HOMO is found to have 4 pairs of 8 lobes which are oriented perpendicular to the molecular axis. ${ }^{19}$ In addition, the shapes of the molecular orbitals are found to be different at 

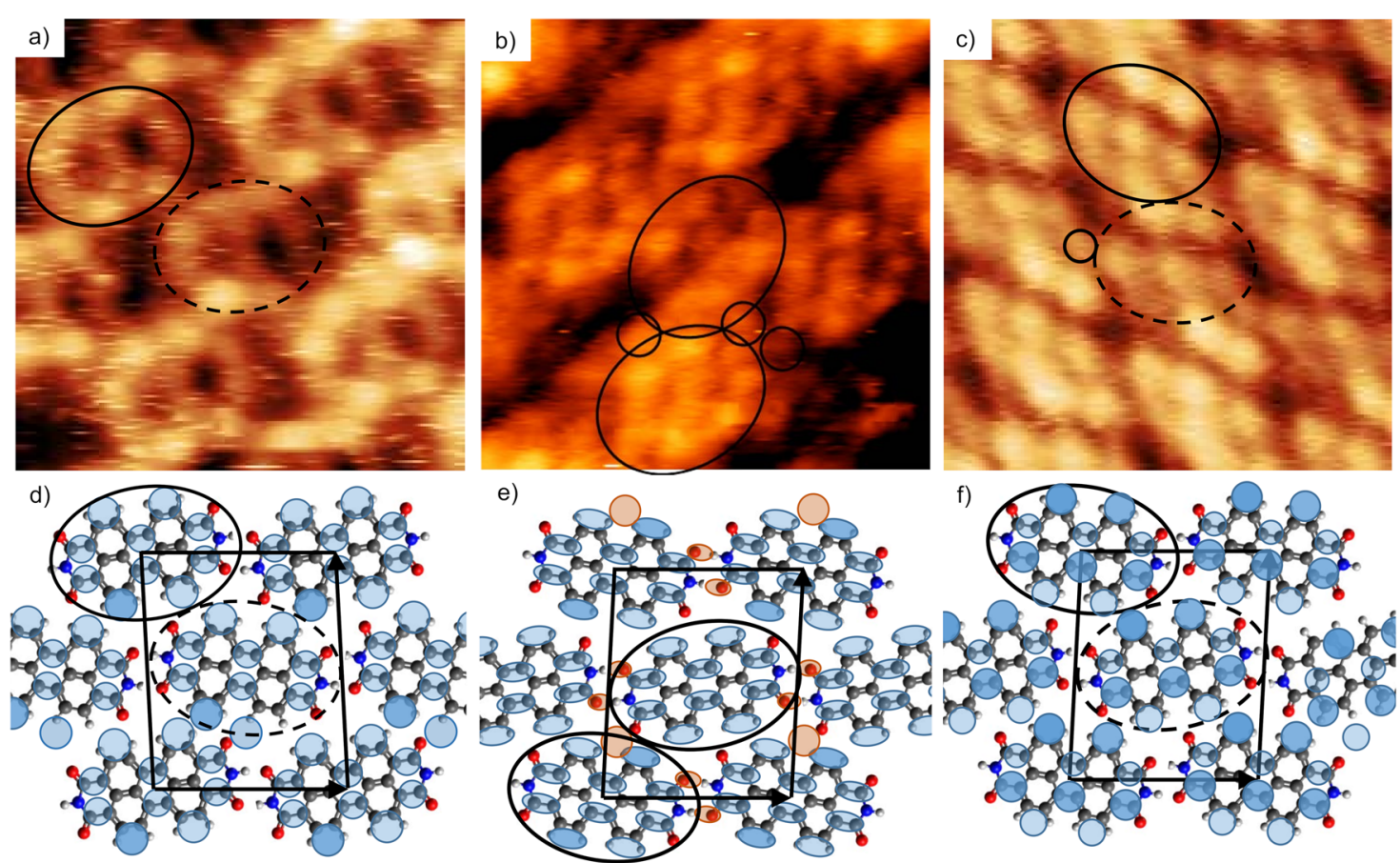

FIG. 2. High resolution filled-state STM images recorded at 1 ML PTCDI coverage at $\mathrm{I}=0.3 \mathrm{nA}$ and biases: (a) $\mathrm{U}=-0.5 \mathrm{~V}$, (b) $\mathrm{U}=-1.0 \mathrm{~V}$, and (c) $\mathrm{U}=-2.0 \mathrm{~V}$. In each STM image, two molecules have been marked with large ovals. Features found beside molecules are marked with smaller circles in (b) and (c). Stick and ball models of (a)-(c), respectively, are shown in (d)-(f), and orbital lobes found in the STM images have been drawn on the molecular models. Lobes on the molecules are drawn in blue, while lobes between molecules have been drawn in red.

different biases. At $-0.5 \mathrm{~V}$ in Fig. 2(a), the 10 orbital lobes are circular in shape and located in such a way that each molecule has two rings of lobes. At $-1.0 \mathrm{~V}$ bias, the lobes have changed their shapes so they are more elongated along the molecular axis and some of them have even shifted in the position along the length of the molecule. Finally at $-2.0 \mathrm{~V}$, the lobes are again more circular but interestingly there is a clear difference in the intensity of the lobes within one molecule. Five of the lobes are very strong and pronounced while the other five are much weaker, especially the three along the center of the molecule are extremely weak.

The general shape of the orbitals of individual molecules does not tell the entire story. This become apparent when looking at molecules in different rows, which have slightly different positions of their lobes and some lobes are more intense than other. Starting with the $-0.5 \mathrm{~V}$ image in Fig. 2(a), it is clear that each molecule has one lobe that is more intense than the other. Furthermore, one of the two rings in the center most molecule (of the unit cell) appears to be broken; i.e., one of the lobes surrounding the hydrogen atoms does not connect with the lobes at the end of the molecule. To fully understand these configurations, the interactions between molecules must be taken into account. The interactions are most easily understood by studying the stick and ball model in Fig. 2(d), where six molecules in three different rows are shown with the orbitals found in the STM. In the upper and lower rows, the lobes of each molecule form two complete rings, with the lower left lobe being brighter than the rest. The molecules in the center row also have a bright lobe at their lower left part, but interestingly one of the two rings appears to be broken because one lobe has moved. This lobe seems to be closer to the oxygen atom of a molecule in an adjacent row. This difference between molecules in different rows is therefore caused by the interactions between the molecules.

Studying the $-1.0 \mathrm{~V}$ bias image in Fig. 2(b) in the same manner reveals further information about the interactions. At this tunneling bias, there are three extra lobes around each molecule that can be observed by STM, and these are marked with smaller rings in Fig. 2(b). Two of the lobes are located where the $\mathrm{N}-\mathrm{H} \cdots \mathrm{O}$ H-bondings are expected to reside. The third lobe is near the location where one of the oxygen atoms, which is not involved in the intra-row interaction, is approaching two of the hydrogen atoms of a neighbouring molecule in an adjacent row. Thus the third lobe should be assigned to a $\mathrm{H} \cdots \mathrm{O} \mathrm{H}$-bonding between molecules in adjacent rows. This last interaction is the same as the one found at $-0.5 \mathrm{~V}$ tunneling bias in Fig. 2(a) where one ring of the central molecule was broken. All the lobes have also been illustrated in the stick and ball model of the unit cell in Fig. 2(e). Because only one extra lobe can be observed between molecules in adjacent rows, this causes an asymmetric behavior of molecules in the unit cell. The $\mathrm{H} \cdots \mathrm{O}$ H-bonding between molecules in adjacent rows is also found in the $-2.0 \mathrm{~V}$ bias image in Fig. 2(c). As shown in Fig. 2(f), it is also clear that the lobes related to the hydrogen that approaches the $\mathrm{O}$ atom forming the $\mathrm{H} \cdots \mathrm{O} \mathrm{H}$ bonding are brighter compared to the other lobes. An electron density plot of the canted structure was presented in a detailed DFT study of various possible structures of PTCDI. ${ }^{7}$ It showed extra densities between molecules that fit well with the results here: Two extra features between molecules along the row and one extra feature on one side of each molecule. ${ }^{7}$ Now these delicate interactions have been clearly observed in STM as 
those extra lobes shown in Fig. 2. Similar intermolecular lobes have also been found in STM between PTCDI molecules on HOPG. $^{8}$

In Fig. 3, two empty-state images are presented in the same way as the filled-state images in Fig. 2. The first emptystate image is recorded at $0.5 \mathrm{~V}$ and is presented in Fig. 3(a) and again two molecules in different rows are marked with circles. In Fig. 3(c), a model containing 6 molecules is presented with the molecular lobes found in STM drawn on them. At the positive sample bias, it is only possible to figure out 8 lobes per molecule which have been marked with blue lobes in Fig. 3(c). These lobes are spread more along the long axis of the molecule but less across the short axis compared to the filled-state images. The four lobes at the center are more intense and appear to be positioned over four of the rings in the perylene core. The two pairs of weaker lobes at the ends of the molecule are located at the carbon atoms in the imide groups. In contrast to the filled-state images, there are no clear lobes surrounding the hydrogen atoms in the empty-state image. However, at one side of the molecules in one type of the row there is a long elongated bright stripe. This stripe is roughly as long as a molecule, and looking closely it is possible to see that this stripe is placed over the four hydrogen atoms of one molecule and two corner parts of its two closest neighbours in the next row. These stripes are marked with yellow ovals in Fig. 3(c). The hydrogen atoms are not clearly represented as specific lobes but are instead seen as a wide stripe involving many hydrogen atoms. Because of this, it is much harder to find more detailed information about the intermolecular interactions in the empty-state image. What can be said though is that the asymmetric configuration, which is found in the filledstate images due to the interaction between hydrogen atoms in one molecule and the oxygen in another, can also be seen in the empty-state image, as the stripe is only being present on one side of the molecules. An image recorded at $1.5 \mathrm{~V}$ bias is presented in Fig. 3(b). The molecular orbital is more similar to the filled-state images here but the molecule still appears to be smaller across the short axis. Seven lobes are found on each molecule. Four of them are positioned on the carbons that bond with the hydrogen atoms, while the other three are sitting on the rings along the core of the molecule. At this bias, it is also possible to find lobes between molecules in the same row.
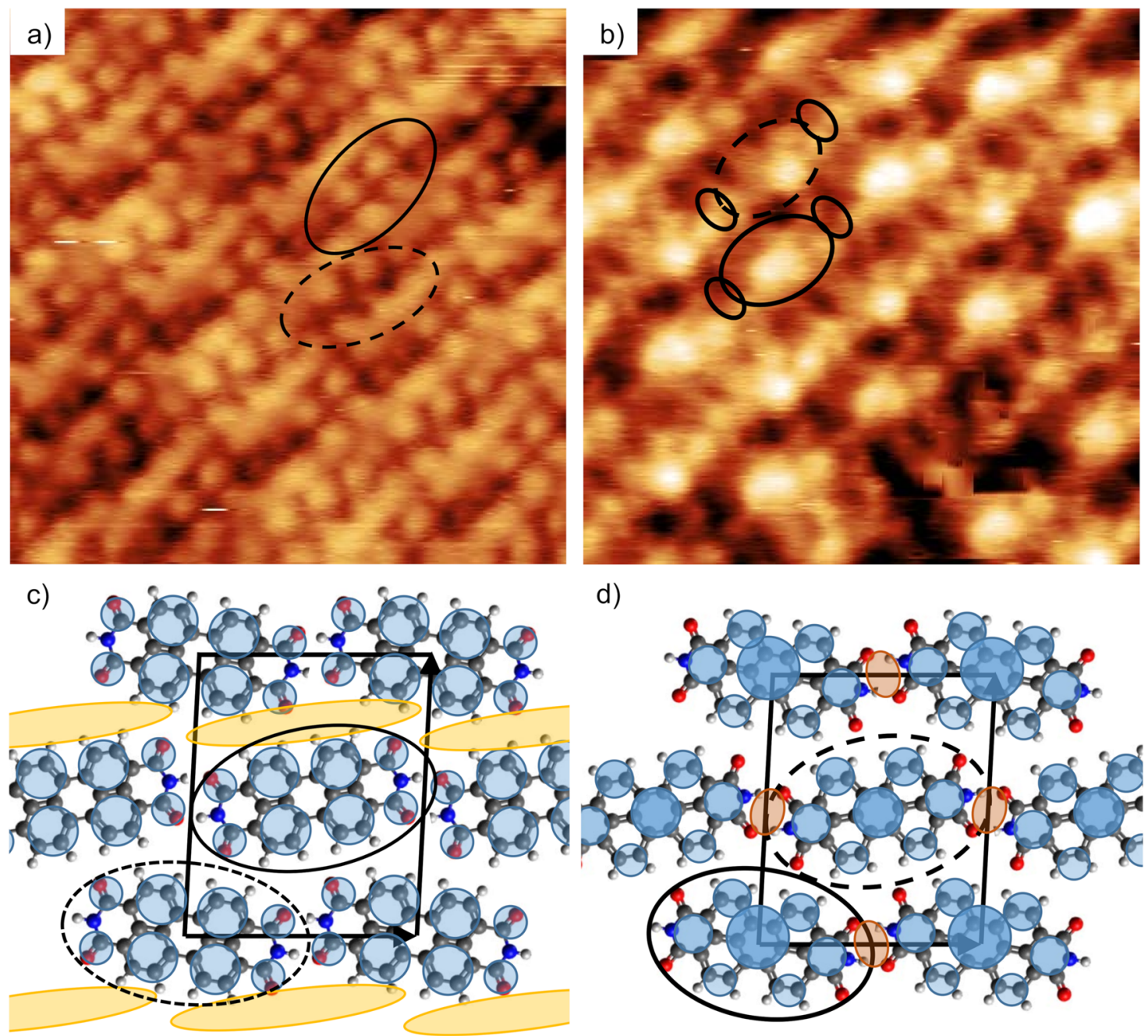

FIG. 3. (a) High resolution empty-state STM images recorded at $1 \mathrm{ML}$ PTCDI coverage at $\mathrm{I}=0.3 \mathrm{nA}$ and bias of $\mathrm{U}=0.5 \mathrm{~V}$. Two molecules in different rows have been marked with circles. (b) Stick and ball model of the molecules with the lobes found in the STM image drawn on the molecules. 
These are marked with small rings in Fig. 3(c) and red ellipses in 3(d). Also, in the rows where the molecules are marked with a non-dashed ellipse in Fig. 3(b) there is a very bright lobe close to the center of the molecule, but offset a bit from its center axis.

It is also interesting to investigate the molecular interaction in the 2 ML film. The structure of the second ML does not have the same superstructure as the first layer and the film is in general more homogeneous. However, at certain tip conditions can a different superstructure with a periodicity of $(2 \sqrt{21} \times(12 / 5) \sqrt{39})$ be observed. High resolution STM images with unit cells in the second ML are presented in Fig. 4. The empty-state image in Fig. 4(a) shows that molecules in different rows have different configurations: One type is dominated by four very bright lobes located around four pairs of hydrogen atoms. The other type shows a strong asymmetric behavior with two bright lobes around two pairs of hydrogen atoms, while two weaker lobes on the opposite side and three small lobes along the perylene core can also be observed. In the filled-state image in Fig. 4(b), the molecular orbitals are quite similar between adjacent rows, but a clear asymmetry in the intensity of the lobes across the molecular axis is observed.
It is also interesting to note that the intensity of the lobes of the middle molecule inside the unit cell is inverted in filled and empty-states: The lobes that are situated on the lower part of the molecules in Fig. 4(a) are comparatively brighter, while they are darker in Fig. 4(b). The asymmetry in intensity that can be observed in Fig. 4 fits very well with the type of interaction that was discussed in Fig. 2. Because only one side of the molecule is interacting with molecules in the adjacent row through a $\mathrm{H} \cdots \mathrm{O}$ H-bonding, one could expect a slight difference in the charge distribution across the molecule. Furthermore, comparing the shape of the filled-state orbital in Fig. 4(b) with the ones in Fig. 2, it is also clear that the interaction with the substrate alters the shapes of the molecular orbitals. The filledstate orbital at $2 \mathrm{ML}$ is very similar to what has been found in single molecular DFT calculations: eight lobes in four pairs, distributed along the molecular axis so there are four lobes on each side of the molecular central axis. ${ }^{19}$

In order to investigate the electronic structures of the PTCDI films, high resolution photoemission has also been carried out. The ARUPS spectra of the clean substrate, with $1 \mathrm{ML}$ and 2 ML PTCDI films recorded at two different angular intervals are presented in Figs. 5 and 6. Figure 5 shows the valence
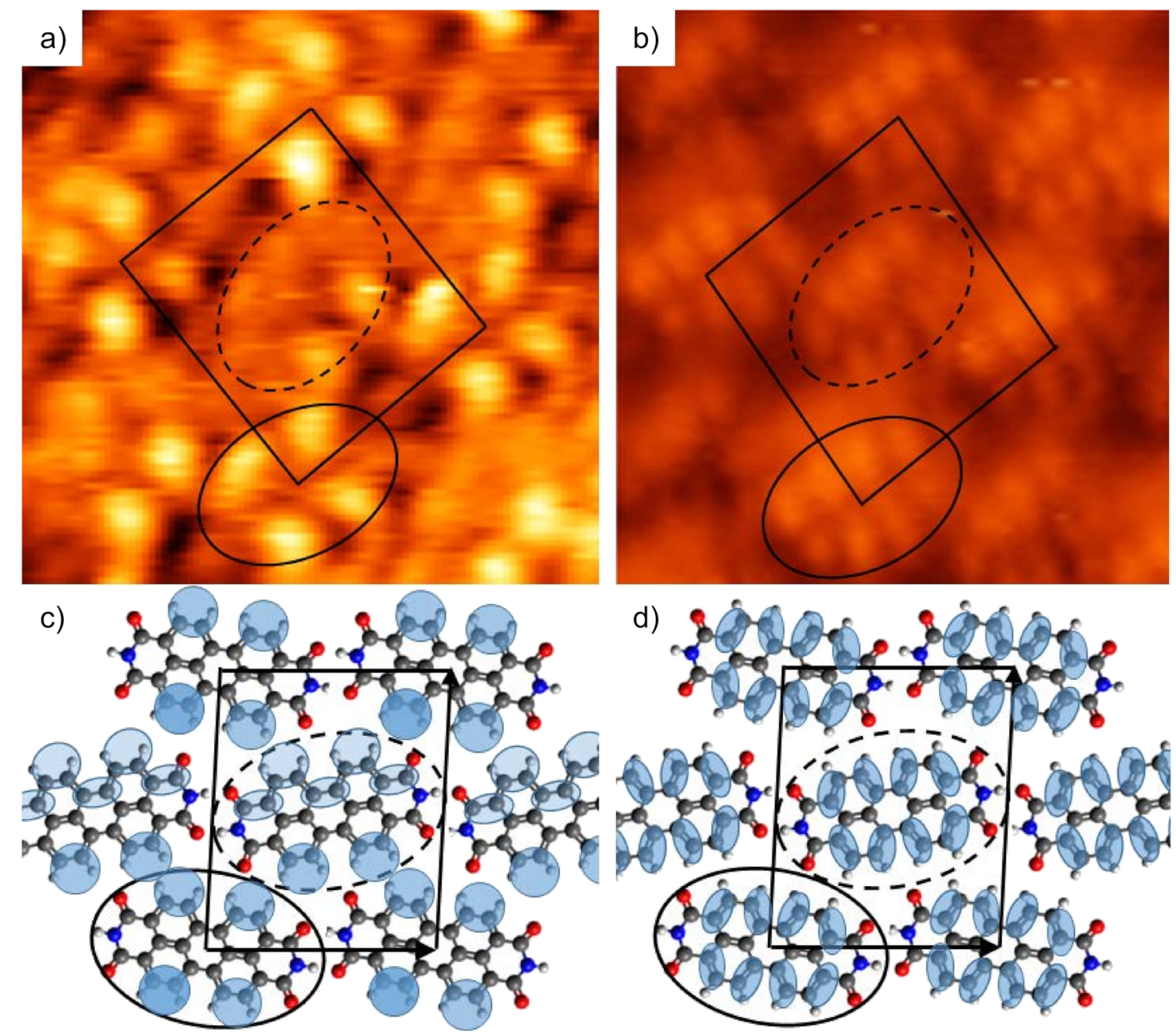

FIG. 4. High resolution STM images recorded at $2 \mathrm{ML}$ PTCDI coverage at $\mathrm{I}=0.3 \mathrm{nA}$ and biases: $(\mathrm{a}) \mathrm{U}=0.5 \mathrm{~V}$ and $(\mathrm{b}) \mathrm{U}=-2.0 \mathrm{~V}$. In each image, two molecules have been marked with solid ovals. Under each image, the observed lobes have been marked on a stick and ball model of the PTCDI molecule. 

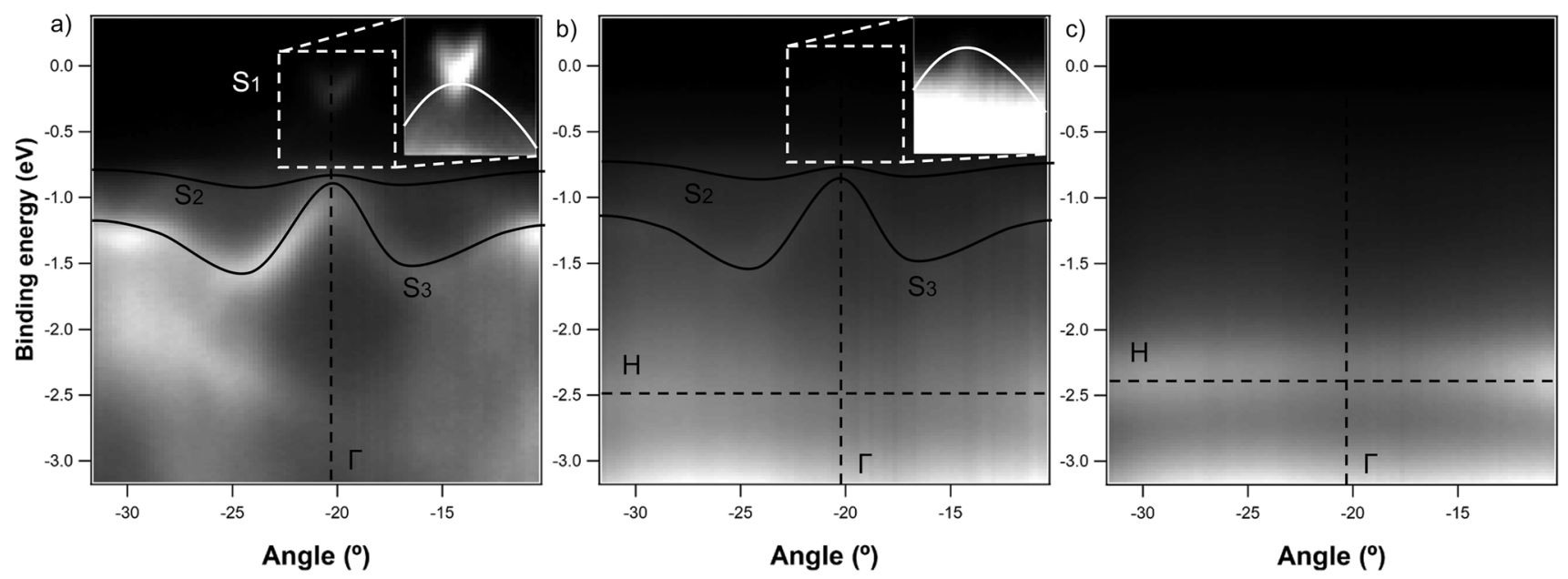

FIG. 5. ARUPS valence band images recorded at $-21^{\circ}$ emission angle in the $\bar{\Gamma}-\bar{M}$ direction, with a photon energy of $40 \mathrm{eV}$ and at PTCDI coverage of (a) 0 ML, (b) $1 \mathrm{ML}$, and (c) 2 ML. Substrate related states are marked with solid lines in (a) and (b), while states related to the PTCDI molecules are marked with dashed lines in (b) and (c). The insets in (a) and (b) show the regions marked with the dashed line in higher contrast.

band images recorded in the $\bar{\Gamma}-\bar{M}$ direction and with angular interval centered at $-21^{\circ}$ emission angle. This is close to the $\bar{\Gamma}$ point of the second $\sqrt{3} \times \sqrt{3}$ surface Brillouin zone, which is located at $-20.1^{\circ}$. The clean surface valence band image, without any molecules is shown in Fig. 5(a), and in it three distinct states are observed and denoted as $S_{1}, S_{2}$, and $S_{3}$. At the $\bar{\Gamma}$ point, $S_{1}$ sits at an energy position of $-0.3 \mathrm{eV}$ relative to the Fermi level, while $S_{2}$ and $S_{3}$ almost merge at the $\bar{\Gamma}$ point at an energy position of $-1.0 \mathrm{eV}$ relative to the Fermi level. These three states are well-known features of the $\mathrm{Ag} / \mathrm{Si}(111)-\sqrt{3} \times \sqrt{3}$ surface. ${ }^{20-22}$ The inset in Fig. 5(a) shows the area around the $\mathrm{S}_{1}$ state with higher contrast. In the inset, a state that reaches up to the $S_{1}$ state has been marked with a white line, which is the upper edge of the bulk valence band. The valence band image in Fig. 5(b) was recorded after $1 \mathrm{ML}$ of PTCDI had been deposited onto the substrate. After the deposition, the states $S_{2}$ and $S_{3}$ are still visible albeit weaker and it is still possible to pinpoint the upper edge of the bulk valance band as illustrated in the inset. All states have been shifted about $0.14 \mathrm{eV}$ toward the Fermi level. As a result, the upper edge of the bulk band is almost touching the Fermi level and the state $S_{1}$ is not found anymore. Except for the shift in energies, there is no change to the clean surface related bands. However, a new state related to the molecules is found at $-2.5 \mathrm{eV}$ and is marked with a dashed line in Fig. 5(b) and denoted as H. Figure 5(c) shows the spectra recorded after another ML of PTCDI was deposited onto the substrate. At 2 ML PTCDI coverage, the surface related states are no longer visible, and the only state that is observed is the $\mathrm{H}$ state that has shifted slightly to $-2.4 \mathrm{eV}$.

The clean surface related bands are fairly strong around the second $\bar{\Gamma}$ point and states related to the molecules are therefore hard to study in the $-21^{\circ}$ valence band images in Fig. 5 . For this reason, valence band images recorded with the angular interval centered at $30^{\circ}$ emission angle are presented in Fig. 6, again for (a) clean substrate, (b) 1 ML-, and (c) 2 ML PTCDI coverage. The clean substrate spectrum in Fig. 6(a) shows the $\mathrm{S}_{2}$ and $\mathrm{S}_{3}$ states away from the $\bar{\Gamma}$ point dispersed at an energy of about $-1 \mathrm{eV}$ below the Fermi level. After deposition of
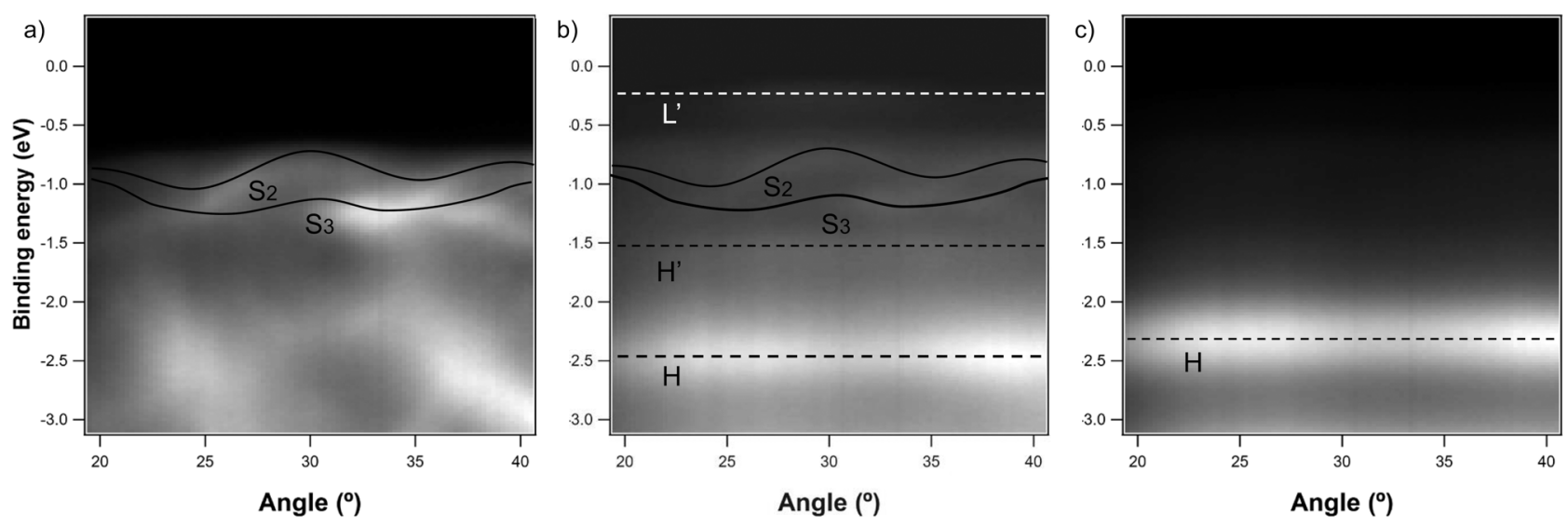

FIG. 6. ARUPS valence band images recorded at $30^{\circ}$ emission angle in the $\bar{\Gamma}-\bar{M}$ direction, with a photon energy of $40 \mathrm{eV}$ and at PTCDI coverage of (a) 0 ML, (b) $1 \mathrm{ML}$, and (c) 2 ML. Substrate related states are marked with solid lines in (a) and (b), while states related to the PTCDI molecules are marked with dashed lines in (b) and (c). 
1 ML PTCDI, the shift toward the Fermi level for the surface related states and the appearance of the molecule-related state $\mathrm{H}$ can be seen in Fig. 6(b). But there are also two other states that are not visible in Fig. 5(b). These two states are located at $-0.3,-1.5 \mathrm{eV}$ with respect to the Fermi level and are tentatively denoted as $\mathrm{L}^{\prime}, \mathrm{H}^{\prime}$, respectively. The states related to the molecules are marked with dashed lines in Fig. 6(b). In the valence band image recorded at 2 ML PTCDI coverage presented in Fig. 6(c), the clean surface related states are no longer visible and only the state $\mathrm{H}$ is found at an energy position of $-2.4 \mathrm{eV}$ below the Fermi level.

In the previous studies, the states close to the Fermi level were preliminary investigated using STS ${ }^{11}$ and angular integrated UPS. ${ }^{14}$ In both studies, the states $\mathrm{L}^{\prime}$ and $\mathrm{H}$ states were found at similar energy positions of around -0.3 and $-2.5 \mathrm{eV}$ relative to the Fermi level at 1 ML PTCDI coverage. The main problem was the $\mathrm{H}^{\prime}$ state that was found at $-1.2 \mathrm{eV}$ in the STS study and at $-1.5 \mathrm{eV}$ in the UPS study. In the UPS study, there is also a state located at about $-1 \mathrm{eV}$ at $1 \mathrm{ML}$ PTCDI coverage. This state was found at the same energy position in the clean substrate and was therefore considered to be a substrate related feature, which might be visible through the first PTCDI layer. It was therefore proposed that the $\mathrm{H}^{\prime}$ state at $-1.2 \mathrm{eV}$ in the STS study was a mix of the surface related state at $-1 \mathrm{eV}$ and the $\mathrm{H}^{\prime}$ state found at $-1.5 \mathrm{eV}$ in UPS.

Using the angular resolved valence band images in Figs. 5 and 6 , it is now very clear that the states around $-1 \mathrm{eV}$ are pure clean surface related states as they keep their dispersions at 1 ML PTCDI coverage. The intermixing of the substrate related states and $\mathrm{H}^{\prime}$ in STS is thus more plausible considering that the substrate related states are stronger at normal emission compared to the states related to the molecules. On the other hand, the substrate related features marked in solid lines in Figs. 5(a) and 5(b) are basically unchanged when the molecules are evaporated onto the substrate. This indicates that the substrate/molecule interaction indeed is weak and hence, the underlying $\sqrt{3} \times \sqrt{3}$ reconstruction is unaltered by the molecules and keeps its electronic structure. Furthermore, the shift of the surface related states toward the Fermi level, is consistent with the shift which was found for the Si $2 p$ core level in the photoemission study. ${ }^{14}$

The state denoted as $\mathrm{H}$ at around $-2.4 \mathrm{eV}$ has previously been found to be the regular HOMO of the PTCDI molecules. ${ }^{19}$ Obviously the interaction with the substrate yields the extra states $\mathrm{H}^{\prime}$ and $\mathrm{L}^{\prime}$. These three filled-states together with the empty-state, L, located at $1.0 \mathrm{eV}$ in STS, are the frontier orbitals at $1 \mathrm{ML}$ PTCDI coverage. Here it is interesting to investigate the electronic structures found in the high resolution STM images with these four states in mind. The regular HOMO state found at $-2.4 \mathrm{eV}$ by ARUPS has been best probed by the $-2.0 \mathrm{~V}$ filled-state image in which each molecule has 10 lobes where half of them were drastically weaker than the rest. The $-1.0 \mathrm{~V}$ filled-state image where extra lobes were found between molecules besides the 10 lobes of each molecule is thus most likely imaging the tail of the $\mathrm{H}^{\prime}$ state located at around $-1.5 \mathrm{eV}$ by ARUPS. The filled-state image recorded at $-0.5 \mathrm{~V}$ where each molecule has two rings of lobes must then be the $\mathrm{L}^{\prime}$ state found close to the Fermi level by ARUPS. The very noisy STM image in Fig. 2(a) indicates that the $\mathrm{L}^{\prime}$ state may not be well-defined for all the molecules. The state with 8 lobes that was probed in the $0.5 \mathrm{~V}$ empty-state image in Fig. 3(a) must originate from the LUMO (L) state, or the tail of it, which was found in the STS study. In a theoretical simulation, there are 8 big lobes in HOMO, while 10 lobes are found in LUMO. ${ }^{19}$ Thus 8 lobes in LUMO could be a result from the half positively charged lobes. This could explain why all the molecules in Fig. 3(a) appear narrower than the normal one. The asymmetric behaviors of 10 lobes in Fig. 3(b) can also be explained in a similar manner.

The formation of the $\mathrm{H}^{\prime}$ and $\mathrm{L}^{\prime}$ states at 1 ML PTCDI coverage has previously been assigned as the results from a HOMO-LUMO split of the molecular orbitals, ${ }^{11}$ similarly to what was observed for the herringbone phase of PTCDA on $\mathrm{Ag} / \mathrm{Si}(111)-\sqrt{3} \times \sqrt{3} .^{23}$ The HOMO-LUMO split in the case of PTCDI was explained to be due to two different adsorption sites for the molecules. This results in these two types having different electronic structures and consequently different frontier orbitals. One type has the states $\mathrm{H}$ and $\mathrm{L}^{\prime}$ as its HOMO and LUMO, while the other type has $\mathrm{H}^{\prime}$ as its HOMO and a LUMO that was found in STS to sit at $1.0 \mathrm{eV}$ above the Fermi level. This means that there are two types of molecules with similar gaps $\left(\mathrm{H} / \mathrm{L}^{\prime}\right.$ and $\left.\mathrm{H}^{\prime} / \mathrm{L}\right)$, i.e., in one of the configurations the LUMO level has been filled and pulled below the Fermi level. In the previous study, the interaction was also investigated using NEXAFS where it was concluded that due to the filling of the LUMO the first transitions to the LUMO are heavily suppressed at PTCDI coverages up to $1 \mathrm{ML} .{ }^{14}$ The changes in NEXAFS spectra and extra states in the valence band close to the Fermi level are similar to what was observed for $1 \mathrm{ML}$ coverage of PTCDA on $\mathrm{Ag}(111)^{24}$ and $\mathrm{Ag}(110){ }^{24,25}$ In those studies, it was also suggested that the changes were due to the LUMO of the molecules being filled or partially filled due to charge donation from the substrate to the molecules. On the other hand, in the core levels for oxygen, carbon, and nitrogen extra features were found on the low binding energy side of the imide related feature for each element at PTCDI coverages up to $1 \mathrm{ML} .{ }^{14}$ These extra features are present because the imide group of some molecules receives more charge and is shifted toward lower binding energy compared to the other molecules.

Looking back at the morphology of the first PTCDI layer, it is possible to distinguish candidates for these two different electron configurations using the superstructure. The superstructure is seen in STM images as rows of darker molecules as those presented in Figs. 1(a) and 1(b). As mentioned above with the two reference points in Fig. 1, one can immediately find that some molecules show exact opposite behavior in contrast. That is, different molecules sitting at the corners of the unit cell switch from dark to bright at two biases when filled and empty-states in the two electron configurations are probed. To be more specific, the $-1.0 \mathrm{~V}$ may detect the partial $\mathrm{H}^{\prime}$ and $\mathrm{L}^{\prime}$ states, while $1.0 \mathrm{~V}$ detects the $\mathrm{L}$ state. The contrast discussed above might originates from the $\mathrm{L}^{\prime}$ and $\mathrm{L}$ states. The inverted brightness thus implies that the $\mathrm{H} / \mathrm{L}^{\prime}$ and $\mathrm{H}^{\prime} / \mathrm{L}$ are located at two types of molecules.

To conclude this part, the changes in the electronic structures with biases fit well with various states found using STS. These observations are thus consistent with the previously 
proposed model for HOMO/LUMO split, which are further strengthened by ARUPS results.

\section{CONCLUSIONS}

The delicate interactions of PTCDI molecules on $\mathrm{Ag} / \mathrm{Si}(111)-\sqrt{3} \times \sqrt{3}$ have been studied using high resolution STM and ARUPS. Different molecular orbital orientations were investigated based on bias-dependent STM images. The intermolecular interactions were found as extra lobes between molecules. Simple models for HOMO and LUMO, together with intermolecular hydrogen-bondings, have been suggested to explain these STM observations. The interaction with the substrate drastically changes the electronic structure of the molecules as the configurations of the molecular orbitals are significantly modified at 1 monolayer compared to ones at higher coverages. This change of the electronic structure is also observed in ARUPS as extra states are found at 1 monolayer coverage. These states observed in the ARUPS spectra are consistent with the STM images. The appearers of extra states are caused by the HOMO/LUMO split. Due to the split, different molecules have different electronic structures, which is in agreement with bias-dependent STM images.

\section{ACKNOWLEDGMENTS}

The work done here was funded by the Swedish Research Council and the Tage Erlander foundation for science and technology.

${ }^{1}$ J. Kwon, M. K. Kim, J.-P. Hong, W. Lee, S. Noh, C. Lee, S. Lee, and J.-I. Hong, Org. Electron. 11, 1288 (2010).

${ }^{2}$ J. Puigdollers, C. Voz, M. Fonrodona, S. Cheylan, M. Stella, J. Andreu, M. Vetter, and R. Alcubilla, J. Non-Cryst. Solids 352, 1778 (2006).

${ }^{3}$ P. Peumans, A. Yakimov, and S. R. Forrest, J. Appl. Phys. 93, 3693 (2003).
${ }^{4}$ V. G. Kozlov, G. Parthasarathy, P. E. Burrows, S. R. Forrest, Y. You, and M. E. Thompson, Appl. Phys. Lett. 72, 144 (1998).

${ }^{5}$ F. S. Tautz, Prog. Surf. Sci. 82, 479 (2007).

${ }^{6}$ J. M. Topple, S. A. Burke, S. Fostner, and P. Grütter, Phys. Rev. B 79, 205414 (2009).

${ }^{7}$ M. Mura, F. Silly, G. A. D. Briggs, M. R. Castell, and L. N. Kantorovich, J. Phys. Chem. C 113, 21840 (2009).

${ }^{8}$ C. Ludwig, B. Gompf, J. Petersen, R. Strohmaier, and W. Eisenmenger, Z. Phys. B: Condens. Matter 93, 365 (1994).

${ }^{9}$ J. C. Swarbrick, J. Ma, J. A. Theobald, N. S. Oxtoby, J. N. O'Shea, N. R. Champness, and P. H. Beton, J. Phys. Chem. B 109, 12167 (2005).

${ }^{10}$ J. Hieulle and F. Silly, J. Mater. Chem. C 1, 4536 (2013).

${ }^{11}$ C. Emanuelsson, H. M. Zhang, E. Moons, and L. S. Johansson, J. Chem. Phys. 146, 114702 (2017).

${ }^{12}$ A. J. Pollard, E. W. Perkins, N. A. Smith, A. Saywell, G. Goretzki, A. G. Phillips, S. P. Argent, H. Sachdev, F. Müller, S. Hüfner, S. Gsell, M. Fischer, M. Schreck, J. Osterwalder, T. Greber, S. Berner, N. R. Champness, and P. H. Beton, Angew. Chem., Int. Ed. 49, 1794 (2010); e-print arXiv:0810.4862.

${ }^{13}$ J. A. Theobald, N. S. Oxtoby, M. A. Phillips, N. R. Champness, and P. H. Beton, Nature 424, 1029 (2003).

${ }^{14}$ C. Emanuelsson, L. S. O. Johansson, and H. M. Zhang, J. Chem. Phys. 149, 044702 (2018).

${ }^{15}$ H. M. Zhang, J. B. Gustafsson, and L. S. O. Johansson, Phys. Rev. B 74, 201304 (2006).

${ }^{16}$ B. N. Jensen, S. M. Butorin, T. Kaurila, R. Nyholm, and L. I. Johansson, Nucl. Instrum. Methods Phys. Res. Sect. A 394, 243 (1997).

${ }^{17}$ P. Butcher and R. I. G. Uhrberg, Phys. World 8, 48 (1995).

${ }^{18}$ A. Ishizaka and Y. Shiraki, J. Electrochem. Soc. 133, 666 (1986).

${ }^{19}$ D. R. T. Zahn, G. N. Gavrila, and M. Gorgoi, Chem. Phys. 325, 99 (2006).

${ }^{20}$ X. Tong, C. Jiang, and S. Hasegawa, Phys. Rev. B 57, 9015 (1998).

${ }^{21}$ H. Aizawa, M. Tsukada, N. Sato, and S. Hasegawa, Surf. Sci. 429, L509 (1999).

${ }^{22}$ H. M. Zhang, K. Sakamoto, and R. I. G. Uhrberg, Phys. Rev. B 64, 245421 (2001).

${ }^{23}$ H. M. Zhang, J. B. Gustafsson, and L. S. O. Johansson, Chem. Phys. Lett. 485, 69 (2010).

${ }^{24}$ Y. Zou, L. Kilian, A. Schöll, T. Schmidt, R. Fink, and E. Umbach, Surf. Sci. 600, 1240 (2006).

${ }^{25}$ L. Kilian, A. Hauschild, R. Temirov, S. Soubatch, A. Schöll, A. Bendounan, F. Reinert, T.-L. Lee, F. S. Tautz, M. Sokolowski, and E. Umbach, Phys. Rev. Lett. 100, 136103 (2008). 